Service social

\title{
Par-delà les frontières. Le travail de groupe et la double problématique du sida et de la consommation de drogues
}

\section{Lawrence Shulman}

Volume 46, numéro 2-3, 1997

Groupes - Symposium 1997

URI : https://id.erudit.org/iderudit/706758ar

DOI : https://doi.org/10.7202/706758ar

Aller au sommaire du numéro

Éditeur(s)

École de service social de l'Université Laval

ISSN

1708-1734 (numérique)

Découvrir la revue

Citer cet article

Shulman, L. (1997). Par-delà les frontières. Le travail de groupe et la double problématique du sida et de la consommation de drogues. Service social, 46(2-3), 37-56. https://doi.org/10.7202/706758ar d'utilisation que vous pouvez consulter en ligne. 


\section{Par-delà les frontières Le travail de groupe et la double problématique du sida et de la consommation de drogues}

Lawrence SHULMAN

À des problèmes complexes il faut souvent apporter des solutions complexes. Le temps est révolu où une seule profession ou encore un modèle de pratique unique pouvait fournir l'aide dont a besoin une grande partie de notre clientèle. Les travailleurs sociaux en travail de groupe admettent depuis longtemps que l'ensemble est plus que la somme de ses parties et qu'une synergie unique se produit quand plus d'une personne est engagée dans le processus d'aide. Le thème du colloque de cette année est donc particulièrement approprié, puisque nous y approfondissons les nombreuses façons dont nous et nos clients pouvons franchir des frontières afin d'accroître l'efficacité de nos pratiques.

Dans cet article, j'aimerais apporter un exemple illustrant le franchissement d'un certain nombre de frontières. II y a quelques années, j'ai enseigné à l'école de service social de l'université de Boston et j'y ai participé avec certains de mes collègues à un programme de développement chapeauté par le CSAP (Center for Substance Abuse Prevention), qui relève du gouvernement fédéral. Durant la deuxième année du programme, chaque professeur a mis en œuvre une expérience individualisée dans son champ de pratique. J'ai alors choisi de travailler au sein d'une agence multiservice de Boston, AIDS Action (Action SIDA), où j'ai coanimé 
un groupe de personnes atteintes du sida qui en étaient au stade préliminaire d'une période de réadaptation après abus de drogues.

John, l'autre coanimateur du groupe, est un membre du personnel à temps plein de l'agence. C'est un conseiller expérimenté en matière d'abus de substances, qui n'est pas travailleur social. Personnellement, en tant que professeur, je travaille d'abord et avant tout dans le domaine de l'enseignement et de la recherche. John est spécialisé en réadaptation, moi pas. II connaît bien les programmes en 12 étapes, comme ceux des Alcooliques anonymes et des Narcotiques anonymes. II s'inspire de la philosophie de l'organisation et s'appuie sur des stratégies de réadaptation. Pour ma part, j'ai aidé à la conception, en travail social, d'un modèle de groupe de soutien basé sur l'entraide, une approche relativement nouvelle pour mon collègue. Disons par ailleurs qu'il est Afro-Américain, alors que je suis Blanc et Juif. En travaillant ensemble nous nous trouvions donc à franchir les frontières que constituent les disciplines professionnelles, la ville et le quartier, l'orientation donnée à la pratique, l'expérience de vie, la race et l'ethnie. Chacune de ces différences soulevait des obstacles que nous devions surmonter, tout en fournissant l'élan nécessaire à notre propre croissance professionnelle et personnelle. Le fait de travailler ensemble, au-delà de ces frontières, a grandement modifié notre pratique et par conséquent l'impact de celle-ci sur les membres de notre groupe.

Les cinq participants du groupe partageaient certains traits, en même temps qu'ils avaient certaines frontières à franchir. Ce qu'ils avaient tous en commun était le fait d'être infectés par le sida, d'entreprendre une réadaptation à la suite d'un abus prolongé de substances diverses (alcool, cocaïne, héroïne, etc.), d'avoir été des sansabri. En outre, la plupart avaient eu recours à la prostitution ou au crime pour payer leur consommation et, ainsi qu'il est apparu durant le groupe, ils avaient tous été victimes d'une forme quelconque d'abus durant leur enfance. II est également ressorti du groupe que les membres avaient tous fait preuve d'une remarquable capacité à réagir aux événements de la vie, capacité que moi, en tant que personne extérieure, je pouvais difficilement évaluer.

Leurs différences ont fait en sorte que chacun avait sa propre vision des frontières qu'il aurait à franchir. Ainsi, pour ces personnes, le début de la guérison se situait entre un jour de complète 
abstinence (un des participants a insisté pour dire que le fait de prendre un verre de temps à temps ne le dérangeait pas) et le début de la deuxième année, qu'une des membres a décrite comme « l'année des sentiments ". C'est l'année, a-t-elle indiqué, où l'on commence à affronter tous les sentiments qu'on fuyait dans la dépendance aux drogues. Selon le modèle du changement par étapes décrit par Prochaska et DiClemente (1986), leur position devant la lutte à leur dépendance allait du " stade contemplatif " (où ils envisageaient la perspective d'apporter des changements dans leur comportement dépendant) au "stade actif " (où ils faisaient des gestes concrets pour vaincre leur accoutumance). Le groupe se réunissait dans un établissement spécialisé offrant un soutien aux personnes autonomes atteintes du sida. Trois des cinq membres vivaient dans cette résidence.

Dans la composition même du groupe, certaines différences créaient également des frontières. Les membres du groupe étaient Kerry, 28 ans, blanc et homosexuel, Tania, 44 ans, transsexuelle, Theresa, 26 ans, hétérosexuelle, Jake, 42 ans, Afro-Américain, et Gerry, 33 ans, Afro-Américain, qui a dû s'absenter du groupe durant deux semaines pour participer à un programme de désintoxication en établissement.

On notait aussi des différences sur le plan de leur lutte contre le "virus ", ainsi qu'ils avaient baptisé le sida. John et Gerry participaient à des essais cliniques où ils recevaient le nouveau traitement contre le sida consistant en une trithérapie. lls avaient montré des baisses significatives de leur charge virale (la quantité de virus VIH dans leur système) et une augmentation tout aussi significative de la numération de leurs cellules $T$, qui mesure la force des anticorps combattant les infections opportunistes susceptibles de les emporter. Pour ces deux membres, l'important était de vivre avec le sida en ayant en tête cette question cruciale posée au cours d'une des séances : « Est-ce que c'est possible? Est-ce que je suis guéri du virus? »

Quant à Theresa, elle attendait que sa charge virale et sa numération de cellules $T$ atteignent le niveau nécessaire pour qu'elle puisse intégrer un groupe expérimentant un médicament. Elle combattait la perte de poids et d'autres symptômes physiques, mais était confiante que la thérapie finirait par inverser le processus de déclin. Kerry refusait de participer au nouveau traitement pharmacologique 
même si un regard sur son état laissait croire qu'il pourrait se qualifier. Tania, après toutes ses années de consommation de drogues et l'usage d'hormones, avait trop de problèmes de santé pour recevoir le nouveau traitement. II s'agissait donc pour elle, comme elle le disait, d'، attendre la mort ", en résistant de son mieux aux drogues afin d'exercer une certaine forme de contrôle sur la période de vie qui lui restait.

Pour les membres du groupe, les frontières à franchir étaient fondées sur le sexe, l'âge, la race, l'orientation sexuelle, sur leur engagement à l'égard de la désintoxication et sur l'étape où ils étaient rendus, sur le stade de leur maladie, leur mode de vie, le pronostic pour chacun et leur volonté de vivre. Ainsi qu'il apparaîtra dans les extraits du compte rendu de l'expérience qui suivent, leurs différences ont créé des obstacles à franchir en même temps qu'elles engendraient l'énergie nécessaire au changement.

\section{SURVOL DES ÉCRITS SUR LE SUJET}

Il y a une dizaine d'années, Googins (1985) identifiait un syndrome qu'il a décrit comme un "désir d'éviter les clients alcooliques » (Avoidance of the Alcoholic Client). II prétendait que, malgré le fait que les " buveurs à problèmes » représentent $12 \%$ de la population ( $21 \%$ des hommes et $5 \%$ des femmes, p. 161), les travailleurs sociaux et les organisations qui les emploient évitaient souvent d'offrir des services à cette clientèle et même de reconnaître le problème chez ces personnes quand il était masqué par d'autres difficultés. Bien que cette négation du problème ait été quelque peu atténuée par des programmes comme celui que chapeaute le CSAP, l'argument de base de Googins demeure vrai.

Dans son souci de comprendre le peu d'empressement à traiter ce problème social particulier, Googins relève un certain nombre de « mythes » entourant l'alcoolisme. Ainsi, des mythes associés à une information lacunaire empêchent plusieurs personnes de comprendre que l'alcool est une drogue qui possède des caractéristiques communes à toutes les drogues. Googins a aussi relevé des mythes « d'attitudes », qui conduisent à une approche moralisatrice selon laquelle les personnes alcooliques sont étiquetées comme moralement « inférieures ». D'autres attitudes, selon Googins, amènent à voir les alcooliques comme des personnes « indésirables, impossibles à traiter et non motivées » (p. 163). 
Certains organismes ont travaillé de longue date pour remédier au manque de traitements dans le champ de l'abus de substances. Qu'on pense aux Alcooliques anonymes (AA), aux Narcotiques anonymes (NA) et à un certain nombre de mouvements, ALANON par exemple, qui s'intéressent plus particulièrement aux familles et aux amis de personnes dépendantes d'une substance. Bien que tous ces groupes puissent différer sous certains aspects, ils possèdent des thèmes communs, dont l'atteinte et le maintien de l'abstinence en aidant d'autres personnes dans le même sens, le recours à une structure bien établie pour contrer l'insécurité ainsi que l'usage de traditions et de devises pour soutenir les efforts de guérison.

Plusieurs de ces groupes décrivent des modèles en 12 étapes qui indiquent la route vers la sobriété. Mon coanimateur et les membres du groupe allaient souvent faire référence à ces étapes comme à des principes directeurs en cas de choix difficiles à faire dans les relations avec les autres. Par exemple, les étapes 8 et 9 donnent aux membres un moyen de vivre avec le sentiment de culpabilité associé à leurs comportements passés "en dressant une liste de toutes les personnes que nous avons lésées et en souhaitant faire amende honorable " ainsi qu'« en réparant directement le tort causé à ces personnes quand c'est possible, sauf si le fait d'agir ainsi risquerait de blesser ces gens ou d'autres personnes ". Ces principes ont été évoqués un soir où les hommes du groupe disaient se sentir coupables d'avoir eu des relations non protégées avec des femmes tout en sachant qu'ils étaient infectés par le virus du sida.

Les AA suivent un modèle fondé sur l'abstinence qui suggère que ce problème est en fait une maladie. Une des nombreuses devises qui guident les membres de l'association vers la guérison est que "Pour un alcoolique, un verre est de trop et un millier ne sont pas assez » (p. 10). Dans les milieux intéressés par l'abus de substance, tout le monde n'accepte pas le modèle " maladie ", ni l'accent mis sur l'abstinence et sur la spiritualité ainsi que d'autres points des programmes en 12 étapes. Cependant, le programme et la philosophie de rétablissement structurés ont été efficaces pour de nombreuses personnes et se sont même révélés déterminants pour trois membres de notre groupe qui assistaient à des réunions des $A A$ et des NA en même temps qu'ils participaient au groupe. En outre, quand le rétablissement d'un des membres semblait compromis, les autres membres du groupe et mon coanimateur recouraient à leur expérience des 12 étapes pour protéger ce rétablissement. 
Matano et Yalom (1991) s'intéressent à la question des frontières à franchir en s'efforçant de synthétiser leur approche " interactionnelle » à la thérapie de groupe et aux méthodes utilisées par les groupes de dépendance chimique traditionnels. Bien qu'ils s'intéressent à un modèle de thérapie de groupe dans lequel les interactions des membres sont mises à profit pour développer l'insight et influencer les changements dans les comportements interpersonnels - un modèle quelque peu différent du groupe de soutien mutuel décrit dans le présent texte -, les principes qu'ils prônent pour une intégration réussie des différentes approches sont pertinents pour notre propos. Les auteurs suggèrent ainsi que :

[...] an interactional approach can be effectively applied to alcoholics if the following guidelines are observed: (1) recovery is always accorded priority, (2) the patient accepts identification as an alcoholic, (3) anxiety is carefully modulated, (4) the proper distinction is made between what the alcoholic is and is not responsible for, (5) the therapist is thoroughly familiar with Alcoholics Anonymous language, steps, and traditions. It is important that therapists not permit misperceptions of AA to be used as therapy resistance and that they be able to harness the wisdom of AA for psychotherapeutic ends (p. 269).

Plusieurs des principes tirés de l'expérience de Matano et Yalom en thérapie de groupe s'appliquent à l'exercice décrit ici et seront soulignés dans le cours de la présentation.

Enfin, Hardesty et Greif (1994) ont décrit les thèmes communs aux groupes de femmes utilisatrices de drogues intraveineuses qui étaient séropositives. Ils ont fait référence à leur travail avec un groupe non volontaire de femmes dans une clinique de maintien à la méthadone.

Ils soulignent l'avantage, propre à un groupe de femmes, qui consiste à pouvoir créer un environnement sûr où il est possible d'aborder des questions liées à la famille d'origine. Les deux femmes du groupe décrit dans cet article ont clairement exprimé leur lutte contre des sentiments de valeur personnelle menant à un cercle vicieux de comportements qui maintenaient et souvent accentuaient la vision négative intériorisée qu'elles avaient d'elles-mêmes. Quand s'ajoute le double stigmate d'abus de substances et d'infection au $\mathrm{VIH}$, on comprend aisément leurs comportements autodestructeurs et on ne peut que s'étonner de leur capacité de résistance. 


\section{LA RÉALISATION DU PROJET}

Dans cette section de l'article je rapporte des extraits des comptes rendus du groupe afin de faire ressortir l'importance du dépassement de frontières à la fois pour les coanimateurs et les membres.

\section{Le stade de formation : décider d'un objectif commun}

Je me suis intéressé ailleurs (Shulman, 1999) aux tâches respectives de l'animateur de groupe et des membres du groupe à la phase initiale. J'ai ainsi suggéré que les membres posent une série de questions à la première séance: Qui sont les animateurs et comment seront-ils dans les rencontres? Quel est le but de ce groupe et est-ce que je perçois un lien quelconque entre le sentiment d'urgence que je ressens et mes besoins actuels? Qui sont les autres membres du groupe et de quel genre de personnes s'agit-il? Ai-je quelque chose en commun avec eux? Les tâches de l'intervenant à la première séance, comme je l'ai déjà mentionné, consistent à : clarifier l'objectif du groupe ainsi que le rôle de l'intervenant; aborder des thèmes liés à l'autorité, comme la confidentialité; inviter les membres à réagir à leur tentative d'établir des liens avec le but du groupe et les autres membres du groupe; faire valoir une philosophie positive de soutien à leur implication dans le groupe.

Pour mon coanimateur, cette notion d'entraide où des membres se soutiennent les uns les autres dans des difficultés particulières - émotives ou cognitives - qui affectent leur vie constitue un concept à la fois nouveau et intéressant. John connaissait surtout le modèle en 12 étapes où, par exemple, une personne présente des expériences liées à l'alcool et aux drogues et où des conférenciers issus du groupe partagent leur sagesse collective des stades du rétablissement ainsi que des stratégies pour maintenir ce rétablissement. L'aide individuelle y est apportée par un conseiller en abus de substances ou par un parrain dans le modèle en 12 étapes; en revanche, l'« échange " y est fortement déconseillé. Dans les rencontres de groupe, il n'est pas jugé approprié d'aider quelqu'un à résoudre des problèmes personnels.

Mes interventions, basées sur mon expérience dans l'animation de groupes de soutien, ont permis à mon coanimateur de voir 
comment l'exploration des sentiments et des connaissances qui influent sur la gestion du quotidien pouvait aider les membres à mieux contrôler leur vie et à assumer leur part de responsabilité dans leurs rapports intimes. Cette prise de pouvoir sur leur vie les a aidés à contrôler leur rétablissement au lieu d'en confier la responsabilité à quelqu'un d'autre.

Par ailleurs, l'expérience personnelle et professionnelle de John, conseiller en matière d'abus de substances, nous a poussés à encourager nos membres à la retenue dans leurs confidences, afin de pouvoir contenir leur anxiété pour ne pas menacer leur rétablissement ou celui d'autres membres qui se trouveraient à un stade différent du processus. Le fait de coanimer avec un conseiller expérimenté en matière d'abus de substances m'a appris à toujours garder bien en tête les questions de rétablissement et à avancer en douceur, là où j'aurais pu pousser plus loin, dans des zones où les membres ne seraient peut-être pas disposés à s'aventurer.

Mais revenons à notre première séance de groupe quand, après une discussion sur des questions de confidentialité, les membres ont commencé à partager leurs expériences de vie, en réponse à notre exposé initial et à notre offre de service. Comme nous avions intentionnellement démarré le groupe juste avant le congé de la l'Action de grâces, il n'était pas surprenant que soient d'abord soulevées les questions liées aux vacances, à la famille, à la solitude et à l'abstinence. Je cherche alors continuellement à atteindre les sentiments sous-tendant les commentaires des membres, alors que John, mon coanimateur, répond aux questions liées au rétablissement. Notre collaboration a commencé par l'influence exercée par chacun sur le travail de groupe ainsi que sur l'autre. L'extrait se poursuit avec ce que j'ai appelé l'échange de problèmes (Shulman, 1999), les membres du groupe partageant leur réaction à notre proposition.

Jake a plongé, disant qu'il voulait raconter ce qui s'était passé durant le week-end. Il était allé chez lui, dans une autre ville de l'État, rendre visite à sa famille. II dit : « J'ai été incapable de rester. Ils ont immédiatement commencé à me rendre fou. Tout le monde là-bas buvait et se droguait et je savais que, si 
je restais, ils m'entraîneraient, et je ne peux pas les laisser faire. Je dois combattre ça. Je sais que je n'ai pas le contrôle de la situation. Si je prends un verre ou me drogue une seule fois, je vais recommencer à aller au bar de huit heures le matin à deux heures la nuit, jusqu'à ce que je sois ivre mort. "

J'ai alors fait remarquer qu'apparemment, en plus de devoir abandonner l'alcool et la drogue, il aurait aussi à abandonner sa famille. Il était d'accord. Kerry dit qu'il était allé dans un bar la semaine précédente et qu'il avait pris deux verres. II a ajouté : " II n'y a pas de problème. Ce n'est pas parce que j'ai pris deux verres que je vais me retrouver au même point que l'année dernière. Je sais bien que vous allez dire que je refuse de voir la réalité en face, mais c'est faux. Je sais que je peux gérer ça. " John, mon coanimateur, dit alors qu'il fallait que nous comprenions que le rétablissement se faisait de façon différente pour chaque personne. Nous devions laisser suffisamment de place à l'intérieur du groupe pour que chacun se sente à l'aise - et non sur la défensive au moment de décrire sa façon de gérer son rétablissement. J'approuvai, et le reste du groupe fit de même.

\section{LE THÈME DE L'AUTORITÉ}

Dans toute première séance, les questions qu'on pose d'abord tournent autour de la relation avec les animateurs, ce que j'ai décrit comme le thème de l'autorité (Shulman, 1999), la lutte continuelle des membres du groupe pour arriver à voir les animateurs comme des symboles d'autorité à la fois exigeants et aidants. Et de fait, au cours de cette première séance, le thème de l'autorité a été soulevé, les membres du groupe ayant besoin de savoir si moi, l'« étranger », j'avais déjà fait usage de drogues et si j'avais participé à un groupe en 12 étapes. Certains membres savaient déjà que mon coanimateur, qui agissait auprès d'eux comme conseiller personnel en ce qui concernait l'abus de drogues, était lui-même en réadaptation, mais on ne savait pas à quoi s'en tenir à mon sujet. La question surgit, comme c'est souvent le cas, d'une façon indirecte.

Tania enchaîna, parlant de toutes les réceptions qui avaient lieu durant le temps des fêtes et de la difficulté qu'elle avait à s'amuser sans avoir d'abord bu ou s'être droguée. "La semaine dernière je suis allée à un party et en regardant les 
gens d'un œil sobre, je me suis dit: "Mon Dieu, est-ce que c'était bien moi? Est-ce que c'est comme ça que je vivais ma vie?" J'ai quitté la fête quand ils ont commencé à se passer la cocaïne, parce que si j'étais restée j'aurais peut-être cédé. Je sais que vous me comprenez tous, étant donné que vous êtes aussi en désintoxication, même si je ne sais pas pour eux " (pointant dans notre direction).

Je profitai de l'occasion pour aborder la question de l'autorité et pour considérer les limites de la confidentialité. Je fis remarquer que Tania avait mentionné à quelques reprises qu'elle s'inquiétait au sujet de la confidentialité et qu'elle avait indirectement posé la question de savoir si nous étions en réadaptation. Je dis que je pourrais parler seulement pour moi. " Non, je ne suis pas en réadaptation; je fais donc figure d'étranger. " J'ajoutai que la question de la confiance qu'ils éprouveraient à mon égard et à l'égard de John aujourd'hui était fondamentale; je pensais aussi qu'ils s'inquiétaient à savoir si un étranger comme moi pouvait comprendre et qu'ils se demandaient ce que nous pourrions partager en dehors du groupe.

Je leur dis que j'étais un travailleur social et que j'enseignais le travail social à l'université. Je leur dis aussi que j'avais demandé à John et à AIDS Action si je pouvais coanimer ce groupe, parce que je trouvais important qu'en tant qu'enseignant je continue à travailler auprès des gens. Je n'étais donc pas un détective de la brigade des stupéfiants et je n'allais pas rapporter leurs paroles ni les dénoncer, sauf s'ils exerçaient des activités illicites à l'intérieur de la résidence, comme de vendre de la drogue, ou s'ils constituaient un danger pour eux ou pour les autres. « J'espère que nous pourrons mériter votre confiance. " Ils approuvèrent en hochant de la tête.

John demanda alors aux membres du groupe s'ils étaient disposés à signer l'entente dont nous avions discuté plus tôt concernant des points comme l'assiduité et la confidentialité. Ils répondirent qu'ils étaient prêts à le faire, et j'ajoutai que nous devrions peut-être signer aussi.

La question de l'autorité allait souvent se poser dans le groupe au cours des séances ultérieures, et cela de plusieurs façons. Une fois les membres en confiance avec moi, et quand je réagissais directement à des signaux donnés de façon indirecte par des membres du groupe, nous explorions ses implications au regard de nos 
différences de genre, de race, d'orientation sexuelle, de classe sociale et de statut. Le thème de l'autorité est demeuré un puissant moteur pour notre travail jusqu'à la dernière séance à laquelle j'ai participé, quand nous avons discuté de la fin de nos rencontres et de ce que le groupe avait signifié pour moi et pour les membres.

\section{LE THÈME DE L'INTIMITÉ : S'AIDER SOI-MÊME EN AIDANT LES AUTRES}

En plus de leur relation avec l'animateur, les membres du groupe devaient aussi travailler leurs rapports entre eux. C'est ce que j'ai décrit ailleurs (Shulman, 1999) sous le « thème de l'intimité ». Bennis et Sheppard (1956) s'appuient sur le conflit pour décider du niveau d'intimité que les membres sont disposés à mettre en jeu, l'ambivalence du groupe se manifestant du fait que certains membres sont " anti-personnels ", c'est-à-dire opposés à toute forme d'intimité, alors que d'autres sont " sur-personnels ", souhaitant donc davantage d'intimité. Dans les extraits qui suivent, Tania exprime d'abord une énergie anti-personnelle, tandis que Theresa tente de mener le groupe à un niveau d'intimité plus grand.

Le conflit était évident autour du désir ou non des membres de parler du sida. Bien que plusieurs thèmes majeurs aient surgi au cours des premières rencontres, les membres du groupe semblaient éviter le sujet du sida. Et quand il était abordé, c'était en passant, les membres changeant rapidement de sujet. Pour reprendre les termes de Bion (1961), ce groupe non formel se tournait vers la «lutte » ou la " fuite " pour éviter la douleur sous-jacente. Par ailleurs, il s'est développé un modèle de fonctionnement selon lequel des membres du groupe exposaient leurs problèmes pendant que les autres écoutaient de façon généralement empathique. L'idée que le groupe puisse se pencher sur le cas particulier d'une personne et lui apporter une aide spécifique leur était étrangère. Alors que les membres recevaient avec sympathie les soucis et les expériences exprimés, ils s'empressaient de donner leur donner leur propre idée sur le sujet. II est apparu qu'ils utilisaient un modèle qui leur était familier, tiré de leur expérience des 12 étapes. Durant les rencontres, mon coanimateur a mis l'accent sur la poursuite de la réadaptation, tandis que je continuais à viser - parfois avec succès, souvent sans - la possibilité d'aide mutuelle. 
La cinquième séance a constitué un point tournant dans le groupe. John et moi avions convenu, à notre dernière rencontre après-groupe, de parler aux membres du fait qu'ils évitaient toute discussion sur le sida et de nous entendre à nouveau avec eux sur le but et la structure du groupe. En assistant au groupe et en nous acceptant comme animateurs, de même qu'en s'acceptant entre eux, les membres du groupe avaient pris ce que j'appellerais la « première décision ". Nous allions maintenant leur demander de prendre la "seconde décision "; ils devraient alors passer de la phase du début à la phase de travail. Cette phase de travail serait caractérisée par un approfondissement de la discussion et par une clarification du sens de l'aide mutuelle. Malheureusement, John était malade le soir de la réunion suivante. Nous nous sommes parlés au téléphone et il était d'accord pour que j'aille de l'avant avec la stratégie convenue.

Kerry, Jake et Tania étaient dans le hall quand je suis arrivé. Tania était mécontente parce que John (mon coanimateur) allait être absent ce soir-là. Elle dit qu'elle avait affaire à lui. Je l'assurai qu'il serait présent à la rencontre de la semaine suivante et que je pouvais lui transmettre un message de sa part. Elle dit: " Je ne pense pas que je vais assister à la réunion. " Elle était vêtue d'un peignoir et avait mauvaise mine. Elle ajouta qu'elle ne se sentait pas bien, mais qu'elle viendrait quand même pour le début de la réunion. Je parlai alors avec Kerry, qui faisait aussi la tête, disant qu'il pouvait venir seulement pour la première demi-heure. Nous avons parlé de ses vacances pendant quelques minutes, jusqu'à ce qu'il se plaigne du groupe, disant que c'étaient des séances de défoulement. II ajouta : " II y a des personnes (et il pointait alors discrètement du doigt vers Tania) qui parlent trop. " Je lui dis que je comprenais ce qu'il voulait dire et que je règlerais ce point le jour même. Pendant ce temps-là, Jake causait avec un autre pensionnaire. II se joignit à nous quand nous commençâmes à monter.

À la réunion, je dis aux membres que si le groupe ne pouvait pas devenir un lieu d'entraide, où ils pouvaient tisser des liens et se soutenir mutuellement, il ne leur apporterait rien. Je leur dis que l'objectif du groupe était d'aider chacun d'eux à trouver la façon de résoudre certaines des difficultés qui survenaient dans leur vie et qu'ils signalaient chaque semaine, 
et non simplement de leur permettre de s'en plaindre. Je dis aussi que je savais qu'il était difficile de faire abstraction de ses propres difficultés pour s'intéresser de près aux problèmes de quelqu'un d'autre, mais c'était le fondement même du groupe. J'ajoutai que je croyais qu'ils éprouvaient tous des difficultés dans leurs tentatives à se rapprocher des gens et que ce groupe était un moyen pour eux de réapprendre à le faire.

Avec enthousiasme Theresa s'exclama que c'était justement cette aide qu'elle attendait du groupe, que c'est de ça qu'elle avait besoin. Elle dit qu'elle voulait parler de son amoureux et des répercussions du VIH sur leurs relations. Pendant qu'elle parlait, Tania l'interrompait, terminant ses phrases à sa place.

Je décidai alors, en mettant à profit la relation positive que je sentais avoir établie avec les membres au cours des quatre premières rencontres, d'arrêter le groupe et de le leur faire remarquer. Je tentai de faire ce que j'appelle une " demande empathique de travail ». En m'opposant directement à Tania, je faisais ressortir ce que je croyais être ses sentiments profonds.

Je dis : «Tania, tu sais que tu n'as pas laissé Theresa terminer une seule phrase? Je me trompe peut-être, mais j'ai l'impression que ces discussions te rendent très nerveuse et que le fait de parler toi-même est un moyen de gérer ton stress. " Elle fait alors : "Ah oui? ", faisant un geste comme si elle se fermait la bouche avec une fermeture-éclair en plus de mettre sa main par-dessus. Je lui dis que j'allais l'aider parce qu'elle avait sûrement beaucoup à donner, mais que je pensais que les autres devaient aussi avoir la chance de s'exprimer. Tania se montra d'accord et, tout le reste de la soirée, elle fit des efforts évidents pour se retenir d'intervenir mal à propos ou d'interrompre les autres.

Chaque fois qu'un membre du groupe soulève un problème général, il y a habituellement un exemple récent qui crée un sentiment d'urgence. J'ai essayé d'aider Theresa à parler davantage de sa "première offre » en recourant à une technique que j'appelle “ aller du général au particulier » (Shulman, 1999).

J'ai demandé à Theresa si quelque chose s'était produit récemment qui la faisait réagir si fortement à cette question. 
Theresa a alors rapporté un incident qui a conduit à une dispute violente avec son petit ami. Ils étaient ensemble en voiture, elle assise derrière. Une autre femme, une amie de son copain, était assise à l'avant. Elle s'est mise à faire ouvertement des avances à son amoureux, sans se soucier le moindrement de Theresa. Très en colère, Theresa a dit que son copain n'a même pas mentionné le fait qu'elle était assise derrière et qu'ils se fréquentaient. Elle trouvait que son ami avait été « irrespectueux » à son égard en ne mettant pas fin aux avances de l'autre femme et en ne tenant pas compte de ce qu'elle pouvait ressentir. (...)

Avec émotion, Tania a alors dit que Theresa était une personne importante et magnifique, qu'elle méritait le respect et que, si elle se respectait elle-même, et Tania croyait que c'était le cas, elle se tiendrait debout et ne laisserait pas ce gars s'en tirer comme ça. Elle devait lui dire directement qu'elle voulait qu'il s'engage envers elle, qu'il la reconnaisse comme son amoureuse. De plus, il ne fallait pas qu'elle garde ce genre de problème en dedans d'elle. Tania dit : " Si tu continues à te mettre ainsi en colère, tout ce que tu arriveras à faire, c'est de te faire mal. Tu vas tomber malade et tu risques même de compromettre ta réadaptation. " Theresa a admis que cela allait lui causer du tort.

Pendant que Theresa nous faisait part d'un problème à la fois très réel et douloureux, elle n'abordait toujours pas la question de son sida, bien qu'elle ait dit au début de cette séance qu'elle souhaitait en parler. J'en étais bien conscient, tout en essayant de comprendre pourquoi elle acceptait la situation actuelle avec son petit ami. Je faisais ce que Schwartz (1961) a décrit comme une « demande de travail » et ce que j'appelle une confrontation facilitante (Shulman, 1999). C'était une demande faite en douceur par laquelle je priais Theresa d'examiner les raisons qui ont fait qu'elle n'a pas approfondi la question.

J'ai voulu savoir pourquoi Theresa avait laissé son copain se défiler quand elle lui a demandé de parler de ses pertes à lui et de son sida à elle. Elle a répondu : « Eh bien, il m'a dit que c'était difficile pour lui d'en parler. » J'ai repris : « Mais tu aurais pu lui demander en quoi c'était si difficile. Pourquoi laisses-tu 
tomber quand il se montre peu disposé à avoir une conversation avec toi? " II y a eu un long silence, puis les traits de Theresa se sont adoucis et elle a dit : " Je suppose que c'est parce qu'au fond je ne veux pas vraiment savoir. " Tout le monde dans la pièce a approuvé de la tête. J'ai dit : "C'est bien, Theresa. Tu fais maintenant preuve de responsabilité. Qu'est-ce que tu as peur d'entendre? "Elle a repris en disant : “ J'ai peur d'être rejetée. »

Jake est alors intervenu, la gorge serrée par l'émotion, disant : "C'est bien ça le problème, quand tu as le virus. Les gens te rejettent. " II s'est alors tourné vers Theresa en lui avouant : " Je peux très bien comprendre pourquoi tu as peur d'être rejetée. Je pense que nous craignons tous la réaction des gens une fois qu'ils savent que nous avons le virus. ”

Theresa a ensuite demandé à Tania si elle la trouvait attirante. II y a eu un silence, puis Tania a dit : " Je trouve que tu es une jeune femme magnifique et je suis certaine que tu pourrais avoir tous les hommes que tu veux. " Theresa a dit qu'elle avait effectivement du succès auprès des hommes. Mais ce qu'elle voulait, c'était une relation sérieuse. Elle dit qu'elle était plus âgée maintenant et qu'elle voulait un véritable engagement avec quelqu'un. Toute la question était là.

Jake, notre membre qui parle peu mais qui n'en pense pas moins, venait de changer la norme et de briser le tabou en soulevant la crainte du rejet associé au sida. La question de Theresa à Tania au sujet de son apparence était indirectement reliée à la crainte du rejet. J'ai tenté de l'aider à s'exprimer en mettant de l'ordre dans ses sentiments.

J'ai dit à Theresa : “ As-tu vraiment peur qu'il pourrait ne pas rester avec toi, que si tu lui parles des autres femmes il pourrait te laisser? » Elle admit que c'est ce qu'elle craignait. Je me suis alors demandé si cela pourrait être utile à Theresa s'ils trouvaient ce qu'elle pourrait dire à son amoureux. Theresa dit que ça l'aiderait parce qu'elle ne savait ni quoi dire ni quand le dire. Elle s'est alors mise à rire en disant : "Peut-être que je pourrais le dire au lit. " Tania a rétorqué : «Oh non! Ne parle pas de ça avant de faire l'amour. Et après 
non plus. » Et j'ai ajouté : «Et n'en parle pas pendant! » Tout le monde a ri et Tania a fait une imitation hilarante de ce que cela donnerait si elle avait une conversation avec le petit ami de Theresa pendant qu'ils font l'amour.

Une fois les rires passés, Tania a dit: « Tu dois trouver un moment tranquille, pas quand vous êtes en pleine dispute, un moment où vous pouvez simplement exprimer vos sentiments. " Tania et moi avons joué les rôles de Theresa et de son ami afin de lui montrer comment faire. (...)

Dans la réponse touchante de Tania à Theresa, nous pouvons reconnaître la dynamique de la "résonance » telle que décrite par Fidele (1994) quand elle parle des groupes de femmes et de la « théorie relationnelle " vue comme un écho et une aptitude à l'empathie.

Je lui ai dit que, si elle l'abordait de front, ce serait très dur pour elle, particulièrement durant les vacances, et que je me demandais qui pourrait la soutenir, en particulier s'il lui disait qu'il ne voulait pas poursuivre leur relation. Theresa a dit qu'elle avait son parrain, et Tania a dit : " Tu m'as, moi aussi. Tu peux m'appeler quand tu veux. » Elle a ajouté : « Je ne réalisais pas quand j'ai commencé à venir à ce groupe qu'il y avait des gens qui avaient vécu des vies comme la mienne, qui éprouvaient les mêmes sentiments que moi, livraient les mêmes combats. Toi, tu es une femme, et tu m'as réellement aidée à comprendre que je n'étais pas la seule dans cette situation. Je vais faire tout ce que je peux pour t'aider. ”

La séance tirant à sa fin, je leur ai demandé ce qu'ils pensaient de la soirée. Je leur ai rappelé que certains membres trouvaient qu'une heure de rencontre c'était trop long, et pourtant nous venions de faire une heure et demie. Theresa a dit que tout le monde l'avait bien aidée. Tania a dit quant à elle : « En fait, je ne voulais pas venir, mais finalement ç'a été très bien. " Jake a dit qu'il avait bien apprécié la soirée et ma façon de mener la rencontre (pour la première fois, il n'a pas distribué de dépliants sur le modèle en 12 étapes).

À la rencontre suivante, dirigée par John et moi, Theresa m'a dit qu'elle avait fait une rechute durant la semaine. Cette confidence a d'abord ébranlé la confiance que j'avais dans mon travail avec le groupe. Je m'en voulais de ne pas avoir équilibré le travail sur les émotions intenses avec le soin à préserver la récupération du membre. 
À cette séance John a donc mis à profit son expérience à faire face aux menaces à la récupération en soutenant Theresa.

Quand je suis arrivé Theresa attendait dans le hall et elle paraissait complètement désemparée. Elle m'a dit qu'elle avait presque consommé durant le week-end (en récupération depuis 18 mois, elle était un modèle pour les autres membres). Elle a dit aussi qu'au lieu de consommer de la drogue elle avait " consommé " un homme. Comme nous étions un peu en avance, j'ai proposé que nous montions à la salle de réunion pour qu'elle me raconte ce qui s'était passé. Theresa s'est assise dans le fauteuil à haut dossier où Tania se trouvait la semaine précédente, en disant: " Je suis à la place de Tania. »

Elle m'a alors appris qu'elle avait rompu avec son petit ami, mais qu'elle l'avait fait d'une façon dont elle n'était pas fière. Quand je lui ai demandé ce qu'elle avait fait, elle m'a répondu qu'elle avait couché avec un autre homme, Jim. Lorsqu'elle avait téléphoné à son ami elle lui avait laissé un message sur son répondeur disant qu'ils se laissaient et aussi qu'elle avait fait l'amour avec quelqu'un d'autre.

Elle a dit qu'elle savait que ce n'était pas ce que nous lui avions suggéré de faire - c'est le moins qu'on puisse dire! -, que Tania lui avait recommandé de ne pas se servir du téléphone, mais bien de s'expliquer en personne, mais elle n'avait pas tenu compte du conseil. Elle a dit qu'elle était tellement désemparée que la toxicomane en elle avait pris le dessus, que la maladie avait pris le dessus, et qu'elle avait eu besoin de consommer. Au lieu de consommer de la drogue, elle s'était tournée vers un homme. Je reconnus que le weekend avait dû être très dur pour elle, et je lui ai demandé si elle croyait qu'elle pourrait en faire part au groupe, parce que je savais que les autres seraient intéressés de savoir ce qui lui était arrivé depuis la dernière rencontre. Elle a dit que oui.

J'ai été très soulagé quand j'ai compris que Theresa n'avait pas fait de rechute par rapport à la drogue ou à l'alcool. J'avais commencé à me sentir coupable à la pensée qu'à la première réunion que j'avais animée seul j'avais pu conduire un des membres à la rechute. J'ai réalisé depuis que je n'avais pas le pouvoir d'aider ni dans ce contexte précis - de nuire à ce point. La récupération de 
Theresa lui appartenait et elle en était à une étape importante du processus.

À $16 \mathrm{~h} 30$ Jake et Tania sont arrivés à leur tour. Tania a dit à Theresa : "Tu es assise à ma place. » J'ai alors dit à Tania que je croyais que Theresa avait besoin de ce fauteuil pour cette semaine. Tania s'est alors assise à côté d'elle et lui a demandé en se penchant vers elle : "Ç’a vraiment été une mauvaise semaine? " Theresa a dit que oui. John, mon coanimateur, a suggéré que nous débutions par trente secondes de silence.

Dans les extraits qui suivent, il est intéressant de noter que cette menace à la réadaptation de Theresa a suscité chez les membres du groupe et chez John de nombreux commentaires fondés sur leur propre expérience. Ils se sont appuyés dans ces réflexions sur diverses métaphores, sur la philosophie, tout en faisant usage de citations tirées de leurs programmes en 12 étapes. J'en suis venu à voir dans ces méthodes des outils efficaces pour fournir une structure nécessaire en situation de crise.

Theresa a raconté son histoire aux autres membres du groupe, la voix remplie d'émotion. Surtout, elle a dit qu'elle se sentait coupable d'avoir agi de cette façon. Elle a dit : « Je peux mettre ça sur le compte de mes sentiments mêlés, de ma dépendance, de ma maladie, mais dans mon cœur je sais que j'ai fait ça pour le punir et je n'y ai pas été de main morte. Je me sens minable. [...] Je l'aime vraiment et maintenant je crois que j'ai tout gâché. Ça me fait beaucoup de peine, surtout avec les fêtes qui arrivent. " Tania, qui s'était tue jusque-là, a demandé à prendre la parole. Se tournant vers Theresa, elle a dit : "Tu as eu tort de le faire par téléphone. C'était une mauvaise façon d'agir, mais tu avais le droit d'être fâchée et, rappelle-toi, c'est lui qui avait commencé. Je sais que c'est dur et que tu as mal agi, mais limportant c'est que tu fais une femme de toi. Tu ne le laisses pas te traiter comme sa pute. II n'était pas prêt à s'engager avec toi. II passait son temps à s'esquiver quand tu essayais d'avoir une conversation avec lui. Alors, c'est peut-être mieux que tu aies provoqué ça maintenant. » John a dit pour sa part : «Tu vis plein d'émotions mêlées ". Et il lui a décrit son ambivalence. 
J'ai fait ressortir le fait que Theresa nous avait dit plusieurs choses. D'un côté, elle s'était affirmée en demandant à son amoureux de la traiter avec respect et en refusant de poursuivre leur relation sans engagement de sa part. D'un autre côté, elle s'y était mal prise. Ce qui était bien, c'est qu'elle n'avait pas consommé de drogue et qu'elle s'était tournée vers ses personnes-ressources (son parrain, les participants aux rencontres), donc, qu'elle avait pris les moyens pour rester en contrôle. Par contre, elle s'était servie d'un gars, ce qu'elle regrettait beaucoup. Theresa a dit : " Je suis fâchée et je me sens coupable, parce que le sexe est comme une drogue pour moi. »

Ainsi que j'en suis venu à le comprendre, les rechutes font partie du processus de récupération. Les membres et les animateurs de groupe ont aidé Theresa à bien saisir que son choix de rechute réduisait les risques pour elle et pour sa récupération. Dans le processus de récupération, l'important est d'apprendre de ses expériences de rechute.

J'ai ensuite rappelé à tout le monde que Theresa nous avait dit la semaine précédente qu'une de ses grandes craintes, si elle perdait son ami, c'était qu'à cause du sida elle n'arriverait pas à trouver un autre homme qui l'aimerait. John a dit : "Tu sais, Theresa, ce que je vois chez toi, c'est beaucoup de progrès. Tu t'es accrochée à tes 18 mois de récupération. Tu n'as pas pris de drogue. Tu étais perturbée, tu avais besoin de quelque chose, mais tu n'as pas lâché. Tu assumes aussi la responsabilité de tes actes, sans chercher à te disculper. "

La réunion s'est poursuivie avec Tania, Jake et Kim rapportant des exemples d'expériences semblables qu'ils avaient vécues et décrivant les répercussions de ces expériences dans leur vie. Ils dirent à nouveau à Theresa que sa réaction était compréhensible, lui rappelant qu'elle avait choisi une rechute moins dommageable. Jake a décrit la façon dont une femme s'était servie de lui pour avoir son frère et comment, en rompant avec elle, il avait aussi rompu avec son frère. II a aussi raconté comment il s'était coupé du monde afin de se protéger :

« J'ai verrouillé ma porte et j'ai coupé la sonnerie du téléphone. Je reste dans ma chambre et je m'isole, parce que je 
sais que si je tente un rapprochement les gens vont encore

me blesser et ça va compromettre ma réadaptation. "

Je dis alors aux membres du groupe qu'il m'apparaissait que c'était tout ou rien avec eux : " Ou bien vous entretenez des relations avec les gens et vous connaissez la douleur et le rejet, surtout à cause du $\mathrm{VIH}$, ou bien vous ne faites rien, vous fermez la porte aux gens, coupez votre téléphone, n'avez pas de relations sexuelles; vous vous retirez, tout simplement. J'ai l'impression que vous avez rarement - sinon jamais - eu de relations avec des gens qui se souciaient de vous et ne vous exploitaient pas. Par conséquent, chez vous, il n'y a pas de juste milieu. »

La réunion s'est achevée et Theresa est partie plus en paix avec elle-même. Je continue à penser que l'aide mutuelle grandit à l'intérieur du groupe et que les membres apprennent à bâtir des relations fondées sur l'empathie, et non sur l'exploitation des autres.

\section{CONCLUSION}

Que m'a finalement rapporté cette expérience de groupe? D’abord, j'y ai appris à respecter davantage d'autres formes d'aide que celles qui m'étaient familières et à me préparer à y recourir dans tous les cas appropriés. J'ai constaté une fois de plus à quel point il est important pour moi de quitter mon costume de professeur afin d'alimenter mon enseignement aux sources du contact direct avec les gens. J'ai aussi observé la façon étonnante dont des personnes différentes à tellement d'égards peuvent traverser leurs propres frontières pour s'aider mutuellement dans leur récupération et dans leur lutte contre une maladie mortelle.

Et, par-dessus tout, j'ai vu à nouveau les ressources immenses que possède notre clientèle même la plus affectée; j'ai vu également la force et l'empathie incroyables dont ces personnes peuvent faire preuve lorsque nous leur fournissons le moyen d'un groupe d'entraide et leur montrons à s'en servir pour se soutenir mutuellement.

Lawrence SHULMAN

Doyen

School of Social Work

University of New York at Buffalo 